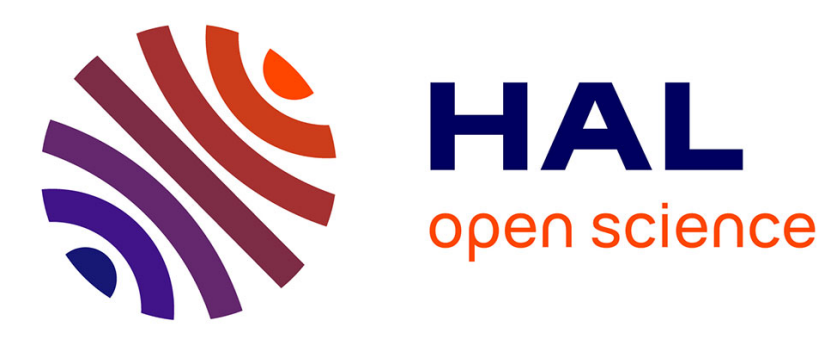

\title{
L-functions of automorphic forms and combinatorics: Dyck paths
}

Laurent Habsieger, Emmanuel Royer

\section{To cite this version:}

Laurent Habsieger, Emmanuel Royer. L-functions of automorphic forms and combinatorics: Dyck paths. Annales de l'Institut Fourier, 2004, 54 (7), pp.2105-2141 (2005). 10.5802/aif.2076 . hal00138563

\section{HAL Id: hal-00138563 \\ https://hal.science/hal-00138563}

Submitted on 14 May 2019

HAL is a multi-disciplinary open access archive for the deposit and dissemination of scientific research documents, whether they are published or not. The documents may come from teaching and research institutions in France or abroad, or from public or private research centers.
L'archive ouverte pluridisciplinaire HAL, est destinée au dépôt et à la diffusion de documents scientifiques de niveau recherche, publiés ou non, émanant des établissements d'enseignement et de recherche français ou étrangers, des laboratoires publics ou privés. 


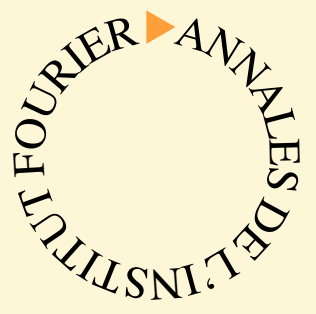

\section{ANNALES}

$\mathrm{DE}$

\section{L'INSTITUT FOURIER}

\section{Laurent HABSIEGER \& Emmanuel ROYER}

\section{$L$-functions of automorphic forms and combinatorics: Dyck paths}

Tome 54, no 7 (2004), p. 2105-2141.

<http://aif.cedram.org/item?id=AIF_2004_54_7_2105_0>

(C) Association des Annales de l'institut Fourier, 2004, tous droits réservés.

L'accès aux articles de la revue «Annales de l'institut Fourier » (http://aif.cedram.org/), implique l'accord avec les conditions générales d'utilisation (http://aif.cedram.org/legal/). Toute reproduction en tout ou partie cet article sous quelque forme que ce soit pour tout usage autre que l'utilisation à fin strictement personnelle du copiste est constitutive d'une infraction pénale. Toute copie ou impression de ce fichier doit contenir la présente mention de copyright.

\section{cedram}

Article mis en ligne dans le cadre du Centre de diffusion des revues académiques de mathématiques http://www.cedram.org/ 


\title{
$L$-FUNCTIONS OF AUTOMORPHIC FORMS AND COMBINATORICS: DYCK PATHS
}

\author{
by Laurent HABSIEGER ${ }^{(*)}$ and Emmanuel ROYER
}

\section{Introduction.}

Since Chowla [Cho34], the behavior of the values at the edge of the critical strip (we shall normalize $L$-functions such that the critical strip is $0<\mathfrak{R} e s<1$ ) of the Dirichlet $L$-functions have been widely studied. Recent results are due to Montgomery \& Vaughan [MV99] and Granville \& Soundararajan [GS02]. The work on these values is motivated by the algebraic interpretation via Dirichlet's class number formula. On the other hand, the study of values at 1 for the higher degree $L$-functions is much more recent. It seems to have begun with the work of Luo [Luo99] who deduced from the study of $L\left(\mathrm{sym}^{2} f, 1\right)$ (when $f$ is a Maass form) results in the deformation theory of modular forms. The experimental study of the values $L\left(\operatorname{sym}^{2} f, 1\right)$ has also been developped by Watkins [Wat02] and Delaunay [Del03].

The Euler product of an higher degree $L$-function being greater than 1 , the corresponding Dirichlet coefficients are not completely multiplicative. It follows that the precise combinatorial behavior of the values at 1 is

$\left(^{*}\right)$ The first author is partially supported by the European Community IHRP Program, within the Research Training Network "Algebraic Combinatorics in Europe", grant HPRN-CT-2001-00272.

Keywords: Symmetric square - modular form - $L$-function - Dyck path - Combinatorics - Narayana number

Math. classification: 11F11 - 11F12 - 11F67 - 11M41 - 05A15 - 05A19 - 11B75 - 11B83 
intricate and actually reveals interesting combinatorial structures. The combinatorial study of the asymptotic negative moments of $f \mapsto L(f, 1)$ and $f \mapsto L\left(\operatorname{sym}^{2} f, 1\right)$ (where $f$ is a primitive form, the parameter being the level of $f$ ) has been done by the second author in [Roy03]. The underlying combinatorial structures were paths in $\mathbb{Z}^{2}$, mainly the Dyck and Riordan paths. Our aim in this paper is to extend the corresponding result to positive moments, and even to positive moments of these values twisted by eigenvalues of Hecke operators. The combinatorial study is more difficult since the asymptotic moments are free of the Möbius function which was fundamental in the proofs in [Roy03]. The combinatorial structures we enlighten are Dyck paths with multivariate statistics (such as return steps, doublerises and last descent steps, see $§ 2)$.

Let us be more explicit. Let $\mu$ be the Möbius function and $P^{-}(N)$ the smallest prime factor of the integer $N$. One defines

$$
\mathcal{N}_{\text {cri }}:=\left\{N \in \mathbb{N}^{*}: \mu(N) \neq 0 \text { and } P^{-}(N) \geqslant \log (3 N)\right\}
$$

Let $H_{k}^{*}(N)$ be the (finite) set of primitive forms of weight $k$ over the group $\Gamma_{0}(N)$ and $\omega(f)$ be the usual harmonic factor (see $\S 1$ for the modular background). One defines

$$
\begin{aligned}
& H_{n}(N):=\sum_{f \in H_{k}^{*}(N)} \omega(f) L(f, 1)^{n} \\
& M_{n}(N):=\sum_{f \in H_{k}^{*}(N)} \omega(f) L\left(\operatorname{sym}^{2} f, 1\right)^{n}
\end{aligned}
$$

for every integer $n \geqslant 0$. We proved in [Roy01] (and actually a more precise result is given in [RW04]) that

$$
\lim _{\substack{N \rightarrow+\infty \\ N \in \mathcal{N}_{\text {cri }}}} M_{n}(N)=M_{n}
$$

where

$$
M_{n}:=\zeta(2)^{n} \sum_{r=1}^{+\infty} \frac{m_{n}(r)}{r}
$$

with

$$
m_{n}(r):=\sum_{\substack{\mathbf{b} \in \mathbb{N}^{n} \\ \operatorname{det} \mathbf{b}=r}} \sum_{\substack{\mathbf{d} \in \mathcal{E}_{n}(\mathbf{b}) \\ \operatorname{det} \mathbf{d}=r}} 1
$$


for every integer $r \geqslant 1$ and

$$
\mathcal{E}_{n}(\mathbf{b}):=\left\{\mathbf{d} \in \mathbb{N}^{n-1}: d_{i} \mid\left(\frac{b_{1} \ldots b_{i}}{d_{1} \ldots d_{i-1}}, b_{i+1}\right)^{2}, \forall i \in[1, n-1]\right\}
$$

for every $\mathbf{b} \in \mathbb{N}^{n}$. We used the following notations: boldfont letters such as $\boldsymbol{\alpha}$ are devoted to vectors; their coordinates are numbered by the index in subscript; the determinant - denoted by det - of a vector is the product of its coordinates; the greatest common divisor of two integers $a$ and $b$ is denoted by $(a, b)$. The same method (see also [CM04]) implies that

$$
\lim _{\substack{N \rightarrow+\infty \\ N \in \mathcal{N}_{\text {cri }}}} H_{n}(N)=H_{n}
$$

where

$$
H_{n}:=\sum_{r=1}^{+\infty} \frac{h_{n}(r)}{r}
$$

with

$$
h_{n}(r):=\sum_{\substack{\mathbf{b} \in \mathbb{N}^{n} \\ \operatorname{det} \mathbf{b}=r}} \sum_{\substack{\mathbf{d} \in \mathcal{F}_{n}(\mathbf{b}) \\ \operatorname{det} \mathbf{d}^{2}=r}} 1
$$

for every integer $r \geqslant 1$ and

$$
\mathcal{F}_{n}(\mathbf{b}):=\left\{\mathbf{d} \in \mathbb{N}^{n-1}: d_{i} \mid\left(\frac{b_{1} \ldots b_{i}}{\left(d_{1} \ldots d_{i-1}\right)^{2}}, b_{i+1}\right), \forall i \in[1, n-1]\right\}
$$

for every $\mathbf{b} \in \mathbb{N}^{n}$. Denoting by $\mathcal{P}$ the set of prime numbers, our first result is

Theorem A. - Let $n$ be a nonnegative integer, then

$$
H_{n+3}=\frac{\zeta(2)^{3 n+3}}{\zeta(4)^{n}} \prod_{p \in \mathcal{P}} s_{n}\left(\frac{p}{p^{2}+1}\right)
$$

and

$$
M_{n+2}=\frac{\zeta(2)^{3 n+3} \zeta(3)^{n}}{\zeta(6)^{n}} \prod_{p \in \mathcal{P}} \ell_{n}\left(-\frac{p}{p^{2}-p+1}\right)
$$

The function $s_{n}$ is a polynomial, related to Narayana numbers. More precisely, let $N_{n}$ be the Narayana polynomial

$$
N_{n}(x)=\sum_{m=0}^{n-1} \frac{1}{n}\left(\begin{array}{c}
n \\
m
\end{array}\right)\left(\begin{array}{c}
n \\
m+1
\end{array}\right) x^{2 m}
$$


then,

$$
N_{n}(x)=\left(1+x^{2}\right)^{n-1} s_{n-1}\left(\frac{x}{1+x^{2}}\right) .
$$

(see lemma 5). The function $\ell_{n}$ is also a polynomial related to Riordan numbers. For every nonnegative integer $m$, define the Riordan number $R_{m}$ of order $m$ by

$$
\sum_{n=0}^{+\infty} R_{n} x^{n}=\frac{2}{1+x+\sqrt{1-2 x-3 x^{2}}}
$$

(see $[$ Roy03, §1.2]) then

$$
\ell_{n}(x)=\sum_{m=0}^{n}(-1)^{m}\left(\begin{array}{c}
n \\
m
\end{array}\right) R_{m} x^{m}
$$

We do remark that the obtained eulerian products are polynomials. The result is similar to the one obtained for negative moments: if $n \geqslant 3$, then

$$
H_{-n}=\frac{\zeta(2)^{n}}{\zeta(4)^{n}} \prod_{p \in \mathcal{P}} s_{n}\left(\frac{p}{p^{2}+1}\right)
$$

and

$$
M_{-n}=\frac{1}{\zeta(2) \zeta(3)^{n}} \prod_{p \in \mathcal{P}} \ell_{n}\left(\frac{p}{p^{2}+p+1}\right)
$$

Using integral representations of the polynomials $s_{n}$ and $\ell_{n}$, we get asymptotic expansions of $H_{n}$ and $M_{n}$.

Theorem B. - Let $n \geqslant 3$ be a integer. Then

$$
\log H_{n}=2 n \log \log n+2 \gamma n+O\left(\frac{n}{\log n}\right)
$$

and

$$
\log M_{n}=3 n \log \log n+3 \gamma n+O\left(\frac{n}{\log n}\right)
$$

Remark that the first term is managable by more elementary (even not obvious) tools - see [Roy01, §3.2.3] - but the combinatorial method has the advantage to give the second term with no additional difficulty and to be more general as applying also to negative moments. 
The way we obtain theorem 1 is to relate the moments to sums of powers. More precisely, let $n$ be a nonnegative integer and $q$ a real number, define

$$
S_{n}(0 ; q):=\sum_{\substack{\left(\alpha_{0}, \ldots, \alpha_{n}\right) \in \mathbb{N}^{n+1} \\ \alpha_{0}=0, \alpha_{n}=0}} \prod_{i=0}^{n-1}\left(q^{\left|\alpha_{2}-\alpha_{2+1}\right|}+\ldots+q^{\alpha_{i}+\alpha_{2+1}}\right)
$$

and

$$
S_{n}^{\prime}(0 ; q):=\sum_{\substack{\alpha \in \mathbb{N}^{n+1} \\ \alpha_{0}=0, \alpha_{n}=0}} \prod_{i=0}^{n-1}\left(q^{\left|\alpha_{\imath}-\alpha_{\imath+1}\right|}+q^{\left|\alpha_{2}-\alpha_{i+1}\right|+2}+\ldots+q^{\alpha_{\imath}+\alpha_{i+1}}\right)
$$

where $\mathbb{N}=\{0,1, \ldots\}$ is the set of nonnegative integers . We show that

$$
M_{n}=\zeta(2)^{n} \prod_{p \in \mathcal{P}} S_{n}\left(0 ; \frac{1}{p}\right)
$$

and

$$
H_{n}=\prod_{p \in \mathcal{P}} S_{n}^{\prime}\left(0 ; \frac{1}{p}\right)
$$

Relating these sums to Dyck paths with statistics "doublerises" and "return step" - see $\S 2$ - we prove the

Proposition A. - Let $n$ be a nonnegative integer and $q$ a real number, then

$$
S_{n+2}(0 ; q)=\frac{\left(1+q^{3}\right)^{n}}{\left(1-q^{2}\right)^{2 n+1}} \ell_{n}\left(-\frac{q}{1-q+q^{2}}\right)
$$

and

$$
S_{n+2}^{\prime}(0 ; q)=\frac{\left(1-q^{4}\right)^{n-1}}{\left(1-q^{2}\right)^{3 n}} s_{n-1}\left(\frac{q}{1+q^{2}}\right)
$$

These results are proved in $\S \S 3.1$ and 4.1.

We next give a unified hypergeometrical formula for the negative and positive moments, valid for both $H_{n}$ and $M_{n}$. Let $a, b$ and $c$ be three 
complex numbers such that $\mathfrak{R} e c>\mathfrak{R} e b>0$, and $z$ a complex number not in the real segment $[1,+\infty[$. One defines

$$
F(a, b, c ; z)=\frac{\Gamma(c)}{\Gamma(b) \Gamma(c-b)} \int_{0}^{1} u^{b-1}(1-u)^{c-b-1}(1-u z)^{-a} \mathrm{~d} u
$$

For every $n \in \mathbb{Z}$ and $\alpha \in\{2,3\}$, define

$$
M_{n, \alpha}= \begin{cases}H_{n} & \text { if } \alpha=2 \\ M_{n} & \text { if } \alpha=3\end{cases}
$$

One then has the

Proposition B. - Let $n \in \mathbb{Z}$ and $\alpha \in\{2,3\}$. Then

$$
M_{n, \alpha}=\prod_{p \in \mathcal{P}}\left[1+\frac{(-1)^{\alpha}}{p}\right]^{-\alpha n} F\left(n, \frac{3}{2}, 5-\alpha ; 4(-1)^{\alpha} \frac{p}{\left[p+(-1)^{\alpha}\right]^{2}}\right)
$$

Remark. - In the case $\alpha=2$, the formula can be simplified in

$$
H_{n}=\prod_{p \in \mathcal{P}} F\left(n, n-1,2 ; p^{-2}\right)
$$

[GR00, 9.134.3].

Finally, we give a combinatorial interpretation of the moments twisted by the eigenvalue $\lambda_{f}(m)$ of the $m$-th Hecke operator (one normalizes the Hecke operators such that $\left|\lambda_{f}(p)\right| \leqslant 2$ for every prime number $\left.p\right)$. Denote by $\mathcal{D}_{n}$ the set of Dyck paths of semilength $n$. If $D \in \mathcal{D}_{n}$, let $\operatorname{RET}(D), \operatorname{DBR}(D)$ and $\mathrm{LD}(D)$ be (respectively) the number of return steps, of doublerises and of last descent steps - see $\S 2$. Then define

$$
K_{n}(x, y, z):=\sum_{D \in \mathcal{D}_{n}} x^{\mathrm{RET}(D)} y^{\mathrm{DBR}(D)} z^{\mathrm{LD}(D)} .
$$

For $q \in] 0,1\left[\right.$ and $\alpha \geqslant 0$, one defines $\Sigma_{n}[\alpha](q)$ by the generating function

$$
\sum_{\alpha=0}^{+\infty} \Sigma_{n}[\alpha](q) t^{\alpha}=\frac{q}{(1-q)\left(1-q^{2}\right) t} K_{n+1}\left(1-q, q^{2}, \frac{\left(1-q^{2}\right) t}{q(1-q t)}\right)
$$

One then has the 
Theorem C. - Let $n$ be a nonnegative integer. Define

$$
\operatorname{Twist}_{2}(n, m)=\prod_{p \mid m} \frac{\Sigma_{n}\left[v_{p}(m) / 2\right]}{\Sigma_{n}[0]}\left(\frac{1}{p}\right) \text {. }
$$

Then

$$
\lim _{\substack{N \rightarrow+\infty \\ N \in \mathcal{N}_{\text {cri }} \\(N, m)=1}} \sum_{f \in H_{k}^{*}(N)} \omega(f) \lambda_{f}(m) L\left(\operatorname{sym}^{2} f, 1\right)^{n+1}=\operatorname{Twist}_{2}(n, m) M_{n+1} .
$$

Similary, define $\sigma_{n}[\alpha](q)$ by

$$
\sum_{\alpha=0}^{+\infty} \sigma_{n}[\alpha](q) t^{\alpha}=\frac{q}{\left(1-q^{2}\right)^{2} t} K_{n+1}\left(1-q^{2}, q^{2}, \frac{\left(1-q^{2}\right) t}{q(1-q t)}\right) .
$$

One then has the

Theorem D. - Let $n$ be a nonnegative integer. Define

$$
\operatorname{Twist}_{1}(n, m)=\prod_{p \mid m} \frac{\sigma_{n}\left[v_{p}(m)\right]}{\sigma_{n}[0]}\left(\frac{1}{p}\right) \text {. }
$$

Then

$$
\lim _{\substack{N \rightarrow+\infty \\ N \in \mathcal{N}_{\text {cri }} \\(m, N)=1}} \sum_{f \in H_{k}^{*}(N)} \omega(f) \lambda_{f}(m) L(f, 1)^{n+1}=\operatorname{Twist}_{1}(n, m) H_{n+1}
$$

Remark. - Motivated by the results of this paper, Cogdell \& Michel developped in [CM04] the analytical view point for the values at 1 of all the symmetric power $L$-functions. Their results show that our theorem 2 extends to higher degrees. They also obtain an interesting probabilistic interpretation.

Acknowledgement. - The authors want to express their gratitude to Étienne Fouvry who had the idea to bring them together. The second author thanks Fabrice Philippe for its valuable comments. 


\section{A skim through $L$-functions.}

The aim of this section is to fix the notations used for modular forms. For more details on the modular background, one refers to $§ 2$ of [RW04]. The space of newforms of weight $k$ and level $N$ is a Hilbert space with respect to the Petersson product

$$
(f, g):=\int_{D_{0}(N)} f(z) \overline{g(z)} y^{k} \frac{\mathrm{d} x \mathrm{~d} y}{y^{2}}
$$

where $D_{0}(N)$ is a fundamental domain of $\Gamma_{0}(N)$. The harmonic weight of $f \in H_{k}^{*}(N)$ is

$$
\omega(f)=\frac{\Gamma(k-1)}{(4 \pi)^{k-1}(f, f)} .
$$

Denote by $H_{k}^{*}(N)$ the (finite) set of primitive forms of weight $k$ and squarefree level $N$. This is the orthogonal basis of the space of newforms of weight $k$ over the modular subgroup $\Gamma_{0}(N)$, consisting of Hecke eigenforms with first Fourier coefficient equal to 1 . The harmonic factor may be considered as an averaging factor since

$$
\lim _{\substack{N \rightarrow+\infty \\ N \in \mathcal{N}_{\text {cri }}}} \sum_{f \in H_{k}^{*}(N)} \omega(f)=1
$$

If $f \in H_{k}^{*}(N)$, one writes its Fourier development as

$$
f(z)=\sum_{n=1}^{+\infty} \lambda_{f}(n) n^{(k-1) / 2} \exp (2 i \pi n z)
$$

If $p$ is a prime number, the coefficient $\lambda_{f}(p)$ admits the decomposition $\lambda_{f}(p)=\alpha_{f}(p)+\beta_{f}(p)$ where $\alpha_{f}(p)$ and $\beta_{f}(p)$ have a norm smaller than 1 (and equal to 1 for all except a finite number of primes).

The $L$ function of a primitive form $f \in H_{k}^{*}(N)$ is defined for $\mathfrak{R} e s>1$ by

$$
L(f, s):=\prod_{p \in \mathcal{P}}\left[1-\frac{\alpha_{f}(p)}{p^{s}}\right]^{-1}\left[1-\frac{\beta_{f}(p)}{p^{s}}\right]^{-1}
$$

Define

$$
L\left(f_{\infty}, s\right):=\pi^{-s} \Gamma\left(\frac{s+(k-1) / 2}{2}\right) \Gamma\left(\frac{s+(k+1) / 2}{2}\right)
$$


then the function

$$
\Lambda(f, s):=L\left(f_{\infty}, s\right) L(f, s)
$$

is entire and satisfies the functional equation

$$
N^{s / 2} \Lambda(f, s)=\varepsilon_{f}(N) N^{(1-s) / 2} \Lambda(f, 1-s)
$$

where $\varepsilon_{f}(N)$ is \pm 1 .

The symmetric square $L$ function of a primitive form $f \in H_{k}^{*}(N)$ is defined for $\mathfrak{R e} s>1$ by

$L\left(\operatorname{sym}^{2} f, s\right):=\prod_{p \in \mathcal{P}}\left[1-\frac{\alpha_{f}(p)^{2}}{p^{s}}\right]^{-1}\left[1-\frac{\alpha_{f}(p) \beta_{f}(p)}{p^{s}}\right]^{-1}\left[1-\frac{\beta_{f}(p)^{2}}{p^{s}}\right]^{-1}$.

Define

$$
L\left(\operatorname{sym}^{2} f_{\infty}, s\right):=\pi^{-3 s / 2} \Gamma\left(\frac{s+1}{2}\right) \Gamma\left(\frac{s+k-1}{2}\right) \Gamma\left(\frac{s+k}{2}\right)
$$

then the function

$$
\Lambda\left(\operatorname{sym}^{2} f, s\right):=L\left(\operatorname{sym}^{2} f_{\infty}, s\right) L\left(\operatorname{sym}^{2} f, s\right)
$$

is entire and satisfies the functional equation

$$
N^{s} \Lambda(f, s)=N^{(1-s)} \Lambda(f, 1-s) .
$$

\section{Dyck paths of statistics (RET, DBR, LD) and Narayana numbers.}

Let $n \geqslant 0$ be an integer. A Dyck path ${ }^{(1)}$ of semilength $n$ is a path in $\mathbb{Z}^{2}$ relying $(0,0)$ to $(n, n)$, with steps $(1,0)$ or $(0,1)$ (one names these steps, respectively horizontal and vertical steps) and never going below the first diagonal. One denotes by $\mathcal{D}_{n}$ the set of Dyck path of semilength $n$. A

(1) Actually, this is the same as what has been untraditionally called "chemin de Catalan de longeur $2 n "$ in [Roy03]. 
Dyck path $D$ is entirely defined by the sequence of abscissas of the starting points of its vertical steps so that there is a bijection between $\mathcal{D}_{n}$ and

$\mathfrak{D}_{n}:=\left\{\left(d_{i}\right)_{i \in[0, n-1]}: 0=d_{0} \leqslant d_{1} \leqslant d_{2} \leqslant \ldots \leqslant d_{n-1}, d_{i} \leqslant i,(i \in[0, n-1])\right\}$

Given $i \in[0, n-1]$, the number $d_{i}$ is then the abscissa of the starting point of the vertical step number $i+1$. The "empty Dyck path" is the point $(0,0)$. One denotes the set consisting of this only path by $\mathcal{D}_{0}$. For $n \geqslant 0$, the number of Dyck paths of semilength $n$ is the $n$th Catalan number, denoted by $C_{n}$.

Let $D$ be a Dyck path of semilength $n$. A doublerise of $D$ is an integer $i$ such that $d_{i}=d_{i+1}$. The number of doublerises of $D$ is then

$$
\operatorname{DBR}(D):= \begin{cases}\#\left\{i \in[0, n-2]: d_{i+1}=d_{i}\right\} & \text { if } n>1 \\ 0 & \text { if } n=1\end{cases}
$$

One also defines RET $(D)$ to be the number of return steps, by what one means vertical steps with starting point on the diagonal,

$\operatorname{RET}(D):=\#\left\{i \in[0, n-1]: d_{i}=i\right\}$ if $n>1$ and $\operatorname{RET}(D):=1$ if $n=1$

Finally, $\mathrm{LD}(D)$ is the number of horizontal steps that end the path $D$, that is

$$
\mathrm{LD}(D)=n-d_{n-1} .
$$

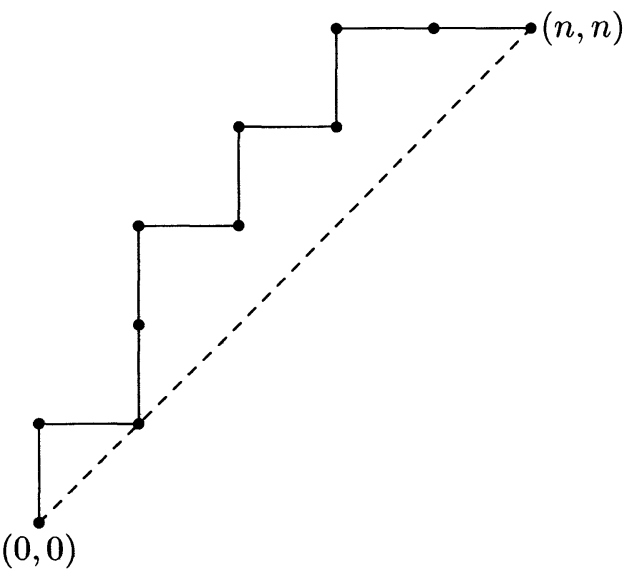

Figure 1. 
We call $\mathrm{LD}(D)$ the number of last descent steps. The empty path is supposed to have DBR, RET and LD all equal to 0. For example, figure 1 represents the Dyck path $D$ of semilength $n=5$, defined by the sequence $(0,1,1,2,3)$. It satisfies $\operatorname{DBR}(D)=1, \operatorname{RET}(D)=2$ and $\operatorname{LD}(D)=2$.

For $n$ a nonnegative integer, one denotes by $K_{n}(x, y, z)$ the generating function of Dyck paths of semilength $n$ and statistics (RET, DBR, LD) and the generating function of these functions is $K(x, y, z ; t)$ :

$$
\begin{aligned}
K_{n}(x, y, z) & :=\sum_{D \in \mathcal{D}_{n}} x^{\mathrm{RET}(D)} y^{\mathrm{DBR}(D)} z^{\mathrm{LD}(D)} \\
K(x, y, z ; t) & :=\sum_{n=0}^{+\infty} K_{n}(x, y, z) t^{n} .
\end{aligned}
$$

The generating function $K(x, y, z ; t)$ is computed in the

Lemma 1. - Let the functions $N, D_{1}$ and $D_{2}$ be defined by

$$
\begin{aligned}
N(x, y, z ; t)=1 & +[1-y-2 x+(2 x-y-1) z] t+(1-y)^{2} z t^{2} \\
+ & {[1+(1-y) z t] \sqrt{1-2(1+y) t+(1-y)^{2} t^{2}} } \\
D_{1}(x, y, z ; t)=1 & +(1-y-2 x) t+\sqrt{1-2(1+y) t+(1-y)^{2} t^{2}}
\end{aligned}
$$

and

$$
D_{2}(x, y, z ; t)=2-\left[1-(1-y) t-\sqrt{1-2(1+y) t+(1-y)^{2} t^{2}}\right] z
$$

then

$$
K(x, y, z ; t)=2 \frac{N(x, y, z ; t)}{D_{1}(x, y, z ; t) D_{2}(x, y, z ; t)} .
$$

Proof. - Let us denote by $b_{n}(d, r, \ell)$ the number of Dyck paths $D \in \mathcal{D}_{n}$ satisfying

$$
\left\{\begin{array}{l}
\operatorname{DBR}(D)=d ; \\
\operatorname{RET}(D)=r ; \\
\operatorname{LD}(D)=\ell
\end{array}\right.
$$


Then

$$
\begin{aligned}
K(x, y, z ; t)=1 & +x \sum_{n=1}^{+\infty} \sum_{d=0}^{+\infty} \sum_{\ell=1}^{+\infty} b_{n}(d, 1, \ell) y^{d} z^{\ell} t^{n} \\
& +\sum_{n=1}^{+\infty} \sum_{d=0}^{+\infty} \sum_{r=2}^{+\infty} \sum_{\ell=1}^{+\infty} b_{n}(d, r, \ell) x^{r} y^{d} z^{\ell} t^{n}
\end{aligned}
$$

Let $D \in \mathcal{D}_{n}$ be a path with $\operatorname{RET}(D) \geqslant 2$. Cutting at the return point of greater abscissa, one sees that this path is the concatenation of a path $D_{1}$ satisfying

$$
\operatorname{RET}\left(D_{1}\right)=\operatorname{RET}(D)-1
$$

and a path $D_{2}$ with $\operatorname{RET}\left(D_{2}\right)=1$ and $\operatorname{LD}\left(D_{2}\right)=\mathrm{LD}(D)-$ see figure 2
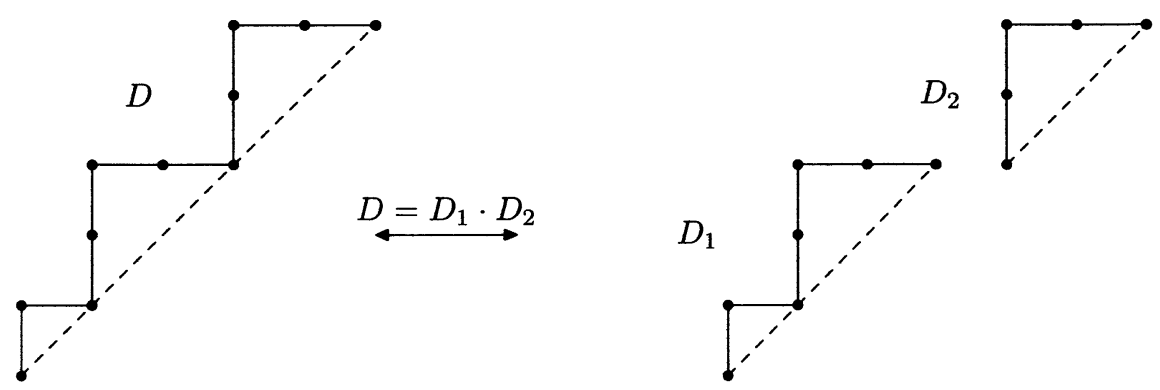

Figure 2.

then obtains,

$$
b_{n}(d, r, \ell)=\sum_{\delta=0}^{+\infty} \sum_{\nu=1}^{+\infty} \sum_{\lambda=1}^{+\infty} b_{\nu}(\delta, r-1, \lambda) b_{n-\nu}(d-\delta, 1, \ell) \quad(r \geqslant 2)
$$

One deduces

(1)

$$
\begin{aligned}
& K(x, y, z ; t)=1+x \sum_{n=1}^{+\infty} \sum_{d=0}^{+\infty} \sum_{\ell=1}^{+\infty} b_{n}(d, 1, \ell) y^{d} z^{\ell} t^{n} \\
& +x\left[\sum_{n=1}^{+\infty} \sum_{d=0}^{+\infty} \sum_{\ell=1}^{+\infty} b_{n}(d, 1, \ell) y^{d} z^{\ell} t^{n}\right]\left[\sum_{n=1}^{+\infty} \sum_{d=0}^{+\infty} \sum_{r=1}^{+\infty} \sum_{\lambda=1}^{+\infty} b_{n}(d, r, \lambda) x^{r} y^{d} t^{n}\right]
\end{aligned}
$$

Let $n \geqslant 2$ and $D$ a path in $\mathcal{D}_{n}$ satisfying $\operatorname{RET}(D)=1$. This path is the concatenation of its first step (necessarily vertical), of a path $D^{\prime}$ in $\mathcal{D}_{n-1}$ 
with $\operatorname{DBR}\left(D^{\prime}\right)=\operatorname{DBR}(D)-1$ and $\operatorname{LD}\left(D^{\prime}\right)=\mathrm{LD}(D)-1$, and of its last step (necessarily horizontal) - see figure 3 . Thus

$$
b_{n}(d, 1, \ell)=\sum_{r=0}^{+\infty} b_{n-1}(d-1, r, \ell-1) \quad(n \geqslant 2)
$$
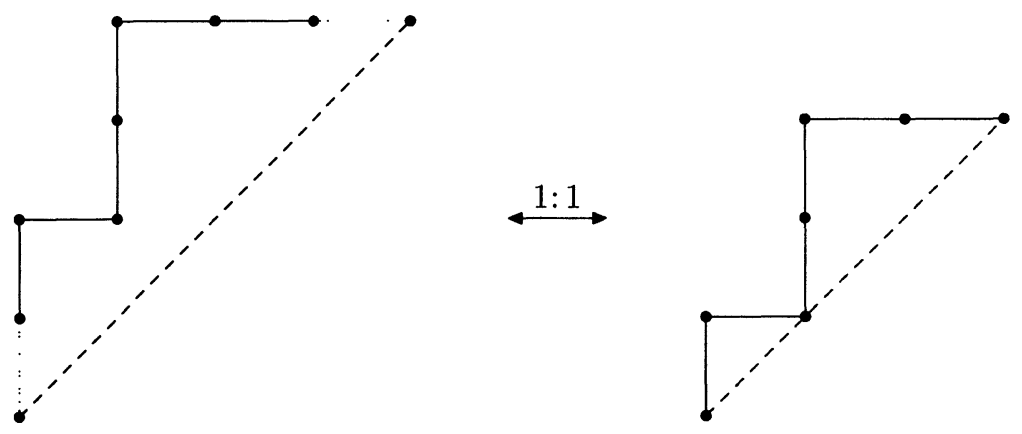

Figure 3.

and

(2)

$$
\sum_{n=1}^{+\infty} \sum_{d=0}^{+\infty} \sum_{\ell=1}^{+\infty} b_{n}(d, 1, \ell) y^{d} z^{\ell} t^{n}
$$

$$
=z t+y z t \sum_{n=1}^{+\infty} \sum_{d=0}^{+\infty} \sum_{r=1}^{+\infty} \sum_{\ell=1}^{+\infty} b_{n}(d, r, \ell) y^{d} z^{\ell} t^{n} .
$$

Reporting (2) in (1) thus gives

(3) $K(x, y, z ; t)=1+[(1-y) x z t+y x z t K(1, y, z ; t)] K(x, y, 1 ; t)$.

Evaluating (3) at $x=1$, one finds

$$
K(1, y, z ; t)=\frac{1+(1-y) z t K(1, y, 1 ; t)}{1-y z t K(1, y, 1 ; t)}
$$

and

$$
K(x, y, z ; t)=\frac{1-y z t K(1, y, 1 ; t)+x z t K(x, y, 1 ; t)}{1-y z t K(1, y, 1 ; t)}
$$


Evaluating (5) at $z=1$ leads then to

$$
K(x, y, 1 ; t)=\frac{1-y t K(1, y, 1 ; t)}{1-x t-y t K(1, y, 1 ; t)}
$$

Reporting (6) in (5) gives

$$
K(x, y, z ; t)=\frac{1+x t(z-1)-y(z+1) t K(1, y, 1 ; t)+y^{2} z t^{2} K(1, y, 1 ; t)^{2}}{[1-y z t K(1, y, 1 ; t)][1-x t-y t K(1, y, 1 ; t)]}
$$

Evaluating (4) at $z=1$ gives a second order equation in $K(1, y, 1 ; t)$ whose solutions are

$$
\frac{1-t+y t \pm \sqrt{1-2 t-2 y t+t^{2}-2 y t^{2}+y^{2} t^{2}}}{2 y t} .
$$

From the convergence of $K(1, y, 1 ; t)$ at $y t=0$, one deduces

$$
K(1, y, 1 ; t)=\frac{1-t+y t-\sqrt{1-2 t-2 y t+t^{2}-2 y t^{2}+y^{2} t^{2}}}{2 y t} .
$$

so that (7) gives the announced expression of $K(x, y, z ; t)$.

For $n$ a nonnegative integer, one denotes by $A_{n}(x, y)$ the generating function of Dyck paths of semilength $n$ and statistics (RET, DBR) and $A(x, y ; t)$ the generating function of these functions:

$$
A_{n}(x, y):=\sum_{D \in \mathcal{D}_{n}} x^{\mathrm{RET}(D)} y^{\mathrm{DBR}(D)}, \quad A(x, y ; t):=\sum_{n=0}^{+\infty} A_{n}(x, y) t^{n}
$$

These functions are specializations at $z=1$ of the preceding ones. One deduces from lemma 1 the

Lemma 2. - One has

$$
A(x, y ; t)=\frac{2-x+x(y-1) t-x \sqrt{1-2(1+y) t+(1-y)^{2} t^{2}}}{2[1-x+x(x+y-1) t]}
$$

As a consequence of lemma 2.1, one has the 
Corollary 3. - Let $n$ be a nonnegative integer and $x$ a real number such that $|x|<1$. Then

$$
A_{n+1}\left(1-x^{2}, x^{2}\right)=\left(1-x^{2}\right) A_{n}\left(1, x^{2}\right)
$$

Proof. - Considering the generating series of both sides, one is led to prove

$$
\frac{1}{t}\left[A\left(1-x^{2}, x^{2} ; t\right)-1\right]=\left(1-x^{2}\right) A\left(1, x^{2} ; t\right)
$$

which is a straightforward consequence of lemma 2 .

One then introduces the special case

$$
N_{n}(x):=A_{n}\left(1, x^{2}\right)
$$

and evaluates it in the

Proposition 4. - Let $n$ be a nonnegative integer and $x$ a real number. Then

$$
N_{n}(x)=\sum_{m=0}^{n-1} \frac{1}{n}\left(\begin{array}{c}
n \\
m
\end{array}\right)\left(\begin{array}{c}
n \\
m+1
\end{array}\right) x^{2 m} .
$$

The number $\frac{1}{n}\left(\begin{array}{c}n \\ m\end{array}\right)\left(\begin{array}{c}n \\ m+1\end{array}\right)$ is the Narayana number of index $(n, m)$. By definition, one has

$$
N_{n}(x)=\sum_{D \in \mathcal{D}_{n}} x^{2 \mathrm{DBR}(D)}
$$

so that, the Narayana number of index $(n, m)$ counts the number of Dyck paths of semilength $n$ with $m$ doublerises. One extracts proposition 2.4 from [Sul98].

Finally, we end the section with an integral expression for the polynomial $N_{n}$.

LemMA 5. - Let $n \geqslant 1$ be a nonnegative integer and $x$ a real number. Define

$$
s_{n}(x)=\frac{2}{\pi} \int_{-\pi / 2}^{\pi / 2}(1+2 x \sin \theta)^{n} \cos ^{2} \theta \mathrm{d} \theta .
$$


Then,

$$
N_{n}(x)=\left(1+x^{2}\right)^{n-1} s_{n-1}\left(\frac{x}{1+x^{2}}\right) .
$$

Proof. - Writing

$$
\sum_{m=0}^{n-1}\left(\begin{array}{c}
n \\
m
\end{array}\right)\left(\begin{array}{c}
n \\
m+1
\end{array}\right) x^{2 m}=
$$

$$
\frac{1}{(n-1) ! x} \sum_{m=0}^{n-1}\left(\begin{array}{c}
n-1 \\
m
\end{array}\right) \frac{n !}{(m+1) !} x^{m+1} \frac{n !}{(n-m) !} x^{m}
$$

gives

$$
\sum_{m=0}^{n-1}\left(\begin{array}{c}
n \\
m
\end{array}\right)\left(\begin{array}{c}
n \\
m+1
\end{array}\right) x^{2 m}=\left.\frac{1}{(n-1) ! x} \frac{\mathrm{d}^{n-1}}{\mathrm{~d} z^{n-1}}(1+x z)^{n}(x+z)^{n}\right|_{z=0}
$$

Then, Cauchy integral formula gives

$$
N_{n}(x)=\frac{1}{2 \pi n x} \int_{0}^{2 \pi}\left(1+x e^{i \theta}\right)^{n}\left(x+e^{i \theta}\right)^{n} e^{-i(n-1) \theta} \mathrm{d} \theta .
$$

Evaluating the real part gives

$$
N_{n}(x)=\frac{1}{2 \pi n x} \int_{0}^{2 \pi}\left(1+2 x \cos \theta+x^{2}\right)^{n} \cos \theta \mathrm{d} \theta .
$$

The result follows then by integration by parts and changes of variables.

3. Moments of $L\left(\operatorname{sym}^{2} f, 1\right)$.

\subsection{A sum of powers related to $L\left(\operatorname{sym}^{2} f, 1\right)$.}

The purpose of this section is to relate the sum

$$
S_{n}(0 ; q):=\sum_{\substack{\alpha \in \mathbb{N}^{n+1} \\ \alpha_{0}=0, \alpha_{n}=0}} \prod_{i=0}^{n-1}\left(q^{\left|\alpha_{\imath}-\alpha_{\imath+1}\right|}+\ldots+q^{\alpha_{\imath}+\alpha_{i+1}}\right)
$$


to Dyck paths. This sum is a specialization of the sum

$$
S_{n}(\alpha ; q):=\sum_{\substack{\alpha \in \mathbb{N}^{n+1} \\ \alpha_{0}=\alpha, \alpha_{n}=0}} \prod_{i=0}^{n-1}\left(q^{\left|\alpha_{\imath}-\alpha_{\imath+1}\right|}+\ldots+q^{\alpha_{i}+\alpha_{i+1}}\right)
$$

which satisfies

$$
S_{1}(\alpha ; q)=q^{\alpha}
$$

and the recursion

$$
S_{n+1}(\alpha ; q)=\sum_{\alpha_{1}=0}^{+\infty} S_{n}\left(\alpha_{1} ; q\right)\left(q^{\left|\alpha-\alpha_{1}\right|}+\ldots+q^{\alpha+\alpha_{1}}\right) .
$$

Let $q \in] 0,1[$ be a real number. One defines an endomorphism on $\mathbb{C}[\alpha]$ by setting, for every integer $r \geqslant 0$,

$$
T_{q}\left(\left(\begin{array}{c}
\alpha \\
r
\end{array}\right)\right):=\sum_{j=0}^{r+1} t_{q, j}(r) \frac{q^{2(r-j)}}{(1-q)\left(1-q^{2}\right)^{r-j+1}}\left(\begin{array}{c}
\alpha \\
j
\end{array}\right)
$$

with

One then has the

$$
t_{q, j}(r):= \begin{cases}1-q & \text { if } j=0 \\ 1 & \text { if } 1 \leqslant j \leqslant r \\ q^{2} & \text { if } j=r+1\end{cases}
$$

Lemma 6. - Let $q \in] 0,1[$ be a real number, for every couple $(r, \alpha)$ of nonnegative integers,

$$
\sum_{\alpha_{1} \in \mathbb{N}} q^{\alpha_{1}}\left(\begin{array}{c}
\alpha_{1} \\
r
\end{array}\right) \sum_{j=\left|\alpha-\alpha_{1}\right|}^{\alpha+\alpha_{1}} q^{j}=q^{\alpha} T_{q}\left(\left(\begin{array}{c}
\alpha \\
r
\end{array}\right)\right)
$$

From this lemma, one deduces the

Lemma 7. - Let $q \in] 0,1[$ be a real number, for every nonnegative integer $n$, there exists a polynomial $\mathfrak{S}_{n, q}$ of degree $n-1$ verifying

$$
\mathfrak{S}_{n+1, q}=T_{q}\left(\mathfrak{S}_{n, q}\right)
$$


such that

$$
S_{n}(\alpha ; q)=q^{\alpha} \mathfrak{S}_{n, q}(\alpha)
$$

for every nonnegative integer $\alpha$.

Proof of lemma 6. - Denoting by $\sum$ the left hand side sum of lemma 6 , one has

$$
\sum=\frac{q^{\alpha}}{1-q}\left[\sum_{\alpha_{1} \geqslant \alpha}\left(\begin{array}{c}
\alpha_{1} \\
r
\end{array}\right) q^{2\left(\alpha_{1}-\alpha\right)}+\sum_{\alpha_{1}<\alpha}\left(\begin{array}{c}
\alpha_{1} \\
r
\end{array}\right)-q \sum_{\alpha_{1}}\left(\begin{array}{c}
\alpha_{1} \\
r
\end{array}\right) q^{2 \alpha_{1}}\right]
$$

Using [GR00, 0.15.1]

$$
\sum_{\alpha_{1}<\alpha}\left(\begin{array}{c}
\alpha_{1} \\
r
\end{array}\right)=\left(\begin{array}{c}
\alpha \\
r+1
\end{array}\right)
$$

one deduces

$$
\sum=\frac{q^{\alpha}}{1-q}\left\{\left(\begin{array}{c}
\alpha \\
r+1
\end{array}\right)+\sum_{\alpha_{1}}\left[\left(\begin{array}{c}
\alpha+\alpha_{1} \\
r
\end{array}\right)-q\left(\begin{array}{c}
\alpha_{1} \\
r
\end{array}\right)\right] q^{2 \alpha_{1}}\right\}
$$

From the Chu-Vandermonde formula

$$
\left(\begin{array}{c}
n+i \\
p
\end{array}\right)=\sum_{r}\left(\begin{array}{c}
n \\
p-r
\end{array}\right)\left(\begin{array}{l}
i \\
r
\end{array}\right)
$$

one obtains

$$
\begin{aligned}
\sum & =\frac{q^{\alpha}}{1-q}\left\{\left(\begin{array}{c}
\alpha \\
r+1
\end{array}\right)+\sum_{\alpha_{1}}\left[(1-q)\left(\begin{array}{c}
\alpha_{1} \\
r
\end{array}\right)+\sum_{j=1}^{r}\left(\begin{array}{c}
\alpha \\
j
\end{array}\right)\left(\begin{array}{c}
\alpha_{1} \\
r-j
\end{array}\right)\right] q^{2 \alpha_{1}}\right\} \\
& =\frac{q^{\alpha}}{1-q}\left[\left(\begin{array}{c}
\alpha \\
r+1
\end{array}\right)+(1-q) \frac{q^{2 r}}{\left(1-q^{2}\right)^{r+1}}+\sum_{j=1}^{r}\left(\begin{array}{c}
\alpha \\
j
\end{array}\right) \frac{q^{2(r-j)}}{\left(1-q^{2}\right)^{r-j+1}}\right]
\end{aligned}
$$

using

$$
\sum_{j}\left(\begin{array}{c}
j+n \\
n
\end{array}\right) z^{j}=\frac{1}{(1-z)^{n+1}}
$$


Proof of lemma 7. - One proceeds by recurrence on $n$. If

$$
S_{n}(\alpha ; q)=q^{\alpha} \sum_{i=0}^{n-1} s(n, q, i)\left(\begin{array}{c}
\alpha \\
i
\end{array}\right)
$$

then, by (10)

$$
\begin{aligned}
S_{n+1}(\alpha ; q) & =\sum_{i=0}^{n-1} s(n, q, i) \sum_{\alpha_{1} \in \mathbb{N}} q^{\alpha_{1}}\left(\begin{array}{c}
\alpha_{1} \\
i
\end{array}\right) \sum_{j=\left|\alpha-\alpha_{1}\right|}^{\alpha+\alpha_{1}} q^{j} \\
& =q^{\alpha} T_{q}\left[\sum_{i=0}^{n-1} s(n, q, i)\left(\begin{array}{c}
\alpha \\
i
\end{array}\right)\right]
\end{aligned}
$$

using lemma 6. The result finally follows from (9).

Corollary 8. - Let $q \in] 0,1[$ be a real number, for every nonnegative integer $n$, one has

$$
S_{n+1}(0, q)=\frac{1}{(1-q)^{n}\left(1-q^{2}\right)^{n}} A_{n}\left(1-q, q^{2}\right)
$$

Proof. - From lemma 6 and (9), one deduces $\mathfrak{S}_{n+1, q}=T_{q}^{n}(1)$. One defines

$$
\Sigma_{n}[\alpha](q):=(1-q)^{n}\left(1-q^{2}\right)^{n} S_{n+1}(\alpha ; q) .
$$

Then

$$
\begin{aligned}
\Sigma_{n}[\alpha](q) & =\sum_{\substack{\left(r_{0}, \ldots, r_{n}\right) \in \mathbb{N}^{n+1} \\
r_{2+1} \leqslant r_{2}+1(0 \leqslant \imath \leqslant n-1) \\
r_{0}=0}}\left(\frac{1-q^{2}}{q^{2}}\right)^{r_{n}}\left[\prod_{i=0}^{n-1} t_{q, r_{2+1}}\left(r_{i}\right)\right]\left(\begin{array}{c}
\alpha \\
r_{n}
\end{array}\right) q^{\alpha} \\
& =\sum_{\substack{\left(r_{0}, \ldots, r_{n}\right) \in \mathbb{N}^{n+1} \\
r_{i+1} \leqslant r_{2}+1(0 \leqslant \imath \leqslant n-1) \\
r_{0}=0}}\left(\frac{1-q^{2}}{q^{2}}\right)^{r_{n}} \times
\end{aligned}
$$




$$
\begin{aligned}
& \times\left[\prod_{\substack{i=0 \\
r_{i+1}=r_{2}+1}}^{n-1} q^{2} \prod_{\substack{i=1 \\
r_{i}=0}}^{n}(1-q)\right]\left(\begin{array}{c}
\alpha \\
r_{n}
\end{array}\right) q^{\alpha} \\
& =\sum_{\substack{\left(r_{0}, \ldots, r_{n}\right) \in \mathbb{N}^{n+1} \\
r_{i+1} \leqslant r_{i}+1(0 \leqslant i \leqslant n-1) \\
r_{0}=0}}\left(\frac{1-q^{2}}{q^{2}}\right)^{r_{n}} \\
& \times q^{2 \#\left\{i \in[0, n-1]: r_{\imath+1}=r_{2}+1\right\}}(1-q)^{\#\left\{i \in[1, n]: r_{2}=0\right\}}\left(\begin{array}{c}
\alpha \\
r_{n}
\end{array}\right) q^{\alpha .}
\end{aligned}
$$

In particular,

$$
\Sigma_{n}[0](q)=\sum_{\substack{\left(r_{0}, \ldots, r_{n}\right) \in \mathbb{N}^{n+1} \\ r_{i+1 \leqslant r_{i}+1(0 \leqslant \imath \leqslant n-1)} \\ r_{0}=r_{n}=0}} q^{2 \#\left\{i \in[0, n-1]: r_{i+1}=r_{i}+1\right\}}(1-q)^{\#\left\{i \in[1, n]: r_{i}=0\right\}}
$$

thus

$$
\begin{aligned}
& \Sigma_{n}[0](q)= \\
& \sum q^{2 \#\left\{i \in[0, n-2]: r_{i+1}=r_{i}+1\right\}}(1-q)^{\#\left\{i \in[0, n-1]: r_{2}=0\right\}} . \\
& \left(r_{0}, \ldots, r_{n-1}\right) \in \mathbb{N}^{n} \\
& r_{i+1} \leqslant r_{i}+1(0 \leqslant i \leqslant n-2) \\
& r_{0}=0
\end{aligned}
$$

Taking $d_{i}=i-r_{i}$, one obtains

$$
\Sigma_{n}[0](q)=\sum_{\substack{\left(d_{0}, \ldots, d_{n-1}\right) \in \mathbb{N}^{n} \\ . d_{0} \leqslant \ldots \leqslant d_{n-1} \\ d_{2} \leqslant 2(0 \leqslant i \leqslant n-2)}} q^{2 \#\left\{i \in[0, n-2]: d_{i+1}=d_{i}\right\}}(1-q)^{\#\left\{i \in[0, n-1]: d_{i}=i\right\}}
$$

the sum being $A_{n}\left(1-q, q^{2}\right)$.

We end this section with the following integral expression of $S_{n+2}(0 ; q)$ :

LEMmA 9. - Let $n$ be a nonnegative integer, then

$$
S_{n+2}(0 ; q)=\frac{\left(1+q^{3}\right)^{n}}{\left(1-q^{2}\right)^{2 n+1}} \ell_{n}\left(-\frac{q}{1-q+q^{2}}\right)
$$


with

$$
\ell_{n}(x):=\frac{4}{\pi} \int_{0}^{\pi / 2}\left(1+x-4 x \sin ^{2} \theta\right)^{n} \cos ^{2} \theta \mathrm{d} \theta
$$

Proof. - Define $F_{n}(q):=(1-q)^{n}\left(1-q^{2}\right)^{n+1} S_{n+2}(0 ; q)$ and

$$
F(q ; t):=\sum_{n=0}^{+\infty} F_{n}(q) t^{n}
$$

The announced equality is equivalent to

$$
F(q ; t)=\sum_{n=0}^{+\infty}\left(1-q+q^{2}\right)^{n} \ell_{n}\left(-\frac{q}{1-q+q^{2}}\right) t^{n}
$$

By corollary 8 , one has

$$
\begin{aligned}
F(q ; t) & =\frac{A\left(1-q, q^{2} ; t\right)-1}{(1-q) t} \\
& =\frac{1}{2 q t}\left(1-\sqrt{1-\frac{4 q t}{1-(1-q)^{2} t}}\right)=\sum_{i=0}^{+\infty} C_{i} \frac{(q t)^{i}}{\left[1-(1-q)^{2} t\right]^{i+1}}
\end{aligned}
$$

for

$$
\sum_{i=0}^{+\infty} C_{i} x^{i}=A(1,1 ; x)=\frac{1}{2 x}(1-\sqrt{1-4 x}) .
$$

By (12), it follows that

$$
\begin{aligned}
F(q ; t) & =\sum_{i=0}^{+\infty} C_{i}(q t)^{i} \sum_{n=i}^{+\infty}\left(\begin{array}{c}
n \\
i
\end{array}\right)(1-q)^{2(n-i)} t^{n-i} \\
& =\sum_{n=0}^{+\infty}\left[\sum_{i=0}^{n}\left(\begin{array}{c}
n \\
i
\end{array}\right) C_{i}(1-q)^{2(n-i)} q^{i}\right] t^{n} \\
& =\sum_{n=1}^{+\infty}\left\{\frac{4}{\pi} \int_{0}^{\pi / 2}\left[(1-q)^{2}+4 q \sin ^{2} \theta\right]^{n} \cos ^{2} \theta \mathrm{d} \theta\right\} t^{n} .
\end{aligned}
$$

The last line (obtained by [Roy03, lemme 6]) gives (14). 


\subsection{Combinatorial expression of the positive moments}

$$
\text { of } L\left(\operatorname{sym}^{2} f, 1\right) \text {. }
$$

In this section, we shall prove the

Proposition 10. - Let $n$ be a nonnegative integer, then

$$
M_{n+2}=\frac{\zeta(2)^{3 n+3} \zeta(3)^{n}}{\zeta(6)^{n}} \prod_{p \in \mathcal{P}} \ell_{n}\left(-\frac{p}{p^{2}-p+1}\right)
$$

By lemma 9, it is equivalent to

LEMmA 11. - Let $n \geqslant 2$ be an integer, then

$$
M_{n}=\zeta(2)^{n} \prod_{p \in \mathcal{P}} S_{n}\left(0 ; \frac{1}{p}\right)
$$

Proof. - By multiplicativity, it suffices to prove

$$
\sum:=\sum_{\nu=0}^{+\infty} \frac{m_{n}\left(p^{\nu}\right)}{p^{\nu}}=S_{n}\left(0, \frac{1}{p}\right) .
$$

One has

$$
\sum=\sum_{\substack{\mathbf{b} \in \mathbb{N}^{n} \\ b_{i} \mid p \infty(1 \leqslant \imath \leqslant n)}} \frac{1}{\operatorname{det} \mathbf{b}} \sum_{\substack{\mathbf{d} \in \mathcal{E}_{n}(\mathbf{b}) \\ \operatorname{det} \mathbf{d}=\operatorname{det} \mathbf{b}}} 1
$$

Defining $\boldsymbol{\alpha} \in \mathbb{N}^{n}$ by

$$
b_{1}=p^{\alpha_{1}}, \quad \frac{b_{1} \ldots b_{i}}{d_{1} \ldots d_{i-1}}=p^{\alpha_{i}} \quad(2 \leqslant i \leqslant n)
$$

and $\boldsymbol{\beta} \in \mathbb{N}^{n}, \boldsymbol{\delta} \in \mathbb{N}^{n-1}$ by

$$
\begin{aligned}
& b_{i}=p^{\beta_{\imath}}, \quad(1 \leqslant i \leqslant n) \\
& d_{i}=p^{\delta_{\imath}}, \quad(1 \leqslant i \leqslant n-1)
\end{aligned}
$$

one gets

$$
\sum=\sum_{\substack{\alpha \in \mathbb{N}^{n} \\ \alpha_{n}=0}} \frac{1}{p^{\alpha_{1}}} \prod_{i=1}^{n-1} \sum_{\substack{\left(\beta_{\imath+1}, \delta_{\imath}\right) \in \mathbb{N}^{2} \\ \beta_{i+1}-\delta_{\imath}=\alpha_{\imath+1}-\alpha_{\imath} \\ \delta_{\imath} \leqslant 2 \min \left(\alpha_{\imath}, \beta_{\imath+1}\right)}} \frac{1}{p^{\beta_{i+1}}}
$$


such that it suffices to prove

$$
\sum_{\substack{\left(\beta_{\imath+1}, \delta_{\imath}\right) \in \mathbb{N}^{2} \\ \beta_{\imath+1}-\delta_{\imath}=\alpha_{\imath+1}-\alpha_{\imath} \\ \delta_{\imath} \leqslant 2 \min \left(\alpha_{\imath}, \beta_{\imath+1}\right)}} \frac{1}{p^{\beta_{\imath+1}}}=p^{-\left|\alpha_{\imath}-\alpha_{\imath+1}\right|}+\ldots+p^{-\left(\alpha_{\imath}+\alpha_{\imath+1}\right)} .
$$

In the case $\alpha_{i+1} \geqslant \alpha_{i}$, equation (17) follows from

$$
\begin{aligned}
\left\{\left(\beta_{i+1}, \delta_{i}\right) \in \mathbb{N}^{2}: \beta_{i+1}-\delta_{i}\right. & \left.=\alpha_{i+1}-\alpha_{i}, \delta_{i} \leqslant 2 \min \left(\alpha_{i}, \beta_{i+1}\right)\right\} \\
& =\left\{\left(\alpha_{i+1}-\alpha_{i}+\delta, \delta\right) \in \mathbb{N}^{2}: \delta \in\left\{0, \ldots, 2 \alpha_{i}\right\}\right\} .
\end{aligned}
$$

In the case $\alpha_{i+1}<\alpha_{i}$, equation (17) follows from

$$
\begin{aligned}
\left\{\left(\beta_{i+1}, \delta_{i}\right)\right. & \left.\in \mathbb{N}^{2}: \beta_{i+1}-\delta_{i}=\alpha_{i+1}-\alpha_{i}, \delta_{i} \leqslant 2 \min \left(\alpha_{i}, \beta_{i+1}\right)\right\} \\
& =\left\{\left(\beta, \alpha_{i}-\alpha_{i+1}+\beta\right) \in \mathbb{N}^{2}: \beta \in\left\{\alpha_{i}-\alpha_{i+1}, \ldots, \alpha_{i}+\alpha_{i+1}\right\}\right\}
\end{aligned}
$$

\subsection{Asymptotic expansion of the positive moments of $L\left(\operatorname{sym}^{2} f, 1\right)$.}

In this section, one proves the

Proposition 12. - Let $n \geqslant 3$, then

$$
\log M_{n}=3 n \log \log n+3 \gamma n+O\left(\frac{n}{\log n}\right) .
$$

Proof. - For $x \leqslant 1 / 3$, one verifies $\ell_{n}(x) \geqslant 0, \ell_{n}^{\prime \prime}(x) \geqslant 0$ and $\ell_{n}^{\prime}(0)=0$ so that $\ell_{n}$ has a minimum on ] $\left.-\infty, 1 / 3\right]$ at 0 . This minimum is $\ell_{n}(0)=1$. One also has $\ell_{n}(x) \leqslant(1-3 x)^{n}$ for $x \leqslant 0$ and

$$
\frac{1+\left(1-t^{2}\right) x}{1-3 x}=1+\frac{\left(4-t^{2}\right) x}{1-3 x} \geqslant 1-\frac{4}{n}
$$

if

$$
-\frac{2}{3} \leqslant x \leqslant-\frac{1}{n}, \quad \text { and } \quad 2 \sqrt{1+\frac{1}{n x}} \leqslant t \leqslant 2 .
$$


If $n \geqslant 5$ and $-2 n \leqslant 3 n x \leqslant-3$ one deduces

$$
\frac{\ell_{n}(x)}{(1+3 x)^{n}} \geqslant \frac{1}{\pi} \int_{2 \sqrt{1+\frac{1}{n x}}}^{2}\left(1-\frac{4}{n}\right)^{n} \frac{2 \mathrm{~d} t}{\sqrt{-n x}}
$$

$$
\geqslant \frac{2}{3125 \pi(-n x)^{-3 / 2}} \geqslant \frac{(-n x)^{-3 / 2}}{4909} .
$$

One the other hand, if $-1 \leqslant n x \leqslant 0$, one has

$$
0 \leqslant \ell_{n}^{\prime \prime}(x) \leqslant 9 n(n-1)\left(1+\frac{3}{n}\right)^{n-2} \ell_{n}(0) \leqslant 181 n^{2}
$$

which gives

$$
\ell_{n}(x) \leqslant 1+91(n x)^{2}
$$

Defining

$$
y_{p}=\frac{p}{p^{2}-p+1},
$$

one has $n y_{p} \geqslant 1$ if and only if $p \leqslant n$. From (18) and (20) one obtains

$$
\prod_{\substack{p \in \mathcal{P} \\ p \leqslant n}}\left(1+3 y_{p}\right)^{n} \prod_{\substack{p \in \mathcal{P} \\ p \leqslant n}}\left[\frac{\left(n y_{p}\right)^{-3 / 2}}{4909}\right] \leqslant \prod_{p \in \mathcal{P}} \ell_{n}\left(-y_{p}\right)
$$

and

$$
\prod_{p \in \mathcal{P}} \ell_{n}\left(-y_{p}\right) \leqslant \prod_{\substack{p \in \mathcal{P} \\ p \leqslant n}}\left(1+3 y_{p}\right)^{n} \prod_{\substack{p \in \mathcal{P} \\ p>n}}\left[1+91\left(n y_{p}\right)^{2}\right] .
$$

As in [Roy03, §2.4.], one then finds

$$
\sum_{p \in \mathcal{P}} \log \left[\ell_{n}\left(-y_{p}\right)\right]=n \sum_{\substack{p \in \mathcal{P} \\ p \leqslant n}} \log \left(1+3 y_{p}\right)+O\left(\frac{n}{\log n}\right)
$$

Writing

$$
1+3 y_{p}=\frac{\left(1-1 / p^{2}\right)^{3}\left(1-1 / p^{3}\right)}{(1-1 / p)^{3}\left(1-1 / p^{6}\right)}
$$

and using Mertens formula [Ten95, théorème 1.11]

$$
\prod_{\substack{p \in \mathcal{P} \\ p \leqslant n}}\left(1-\frac{1}{p}\right)^{-1}=e^{\gamma} \log n\left[1+O\left(\frac{1}{\log n}\right)\right]
$$


one obtains

$$
\sum_{\substack{p \in \mathcal{P} \\ p \leqslant n}} \log \left(1+3 y_{p}\right)=3 \log \log n+3 \gamma+\log \frac{\zeta(6)}{\zeta(2)^{3} \zeta(3)}+O\left(\frac{1}{\log n}\right) .
$$

Reporting this expansion in (21), one finally finds

$$
\log M_{n}=3 n \log \log n+3 \gamma n+O\left(\frac{n}{\log n}\right) .
$$

4. Moments of $L(f, 1)$.

\subsection{A sum of powers related to $L(f, 1)$.}

Consider

$$
S_{n}^{\prime}(\alpha ; q):=\sum_{\substack{\alpha \in \mathbb{N}^{n+1} \\ \alpha_{0}=\alpha, \alpha_{n}=0}} \prod_{i=0}^{n-1}\left(q^{\left|\alpha_{\imath}-\alpha_{\imath+1}\right|}+q^{\left|\alpha_{\imath}-\alpha_{\imath+1}\right|+2}+\ldots+q^{\alpha_{\imath}+\alpha_{\imath}+1}\right)
$$

The aim of this section is to relate $S_{n}^{\prime}(0 ; q)$ to Dyck paths. One proves the

Proposition 13. - Let $q \in] 0,1[$ be a real number, for every nonnegative integer $n$, one has

$$
S_{n+2}^{\prime}(0 ; q)=\frac{1}{\left(1-q^{2}\right)^{2 n+2}} A_{n+1}\left(1-q^{2}, q^{2}\right)=\frac{1}{\left(1-q^{2}\right)^{2 n+1}} N_{n}(q)
$$

The second equality of proposition 13 is a consequence of the corollary 3 and of the definition (8). The proof of the first equality is the same as the one given for corollary 8 in $\S 3.1$. The only significant difference is that one replaces $T_{q}$ by $T_{q}^{\prime}$ defined by

$$
T_{q}^{\prime}\left(\left(\begin{array}{c}
\alpha \\
r
\end{array}\right)\right):=\sum_{j=0}^{r+1} t_{q, j}^{\prime}(r) \frac{q^{2(r-j)}}{\left(1-q^{2}\right)^{r-j+2}}\left(\begin{array}{c}
\alpha \\
j
\end{array}\right)
$$


with

$$
t_{q, j}^{\prime}(r):= \begin{cases}1-q^{2} & \text { if } j=0, \\ 1 & \text { if } 1 \leqslant j \leqslant r, \\ q^{2} & \text { if } j=r+1 .\end{cases}
$$

Lemma 6 has then to be replaced by the following one

Lemma 14. - Let $q \in] 0,1[$ be a real number, for every couple $(r, \alpha)$ of nonnegative integers,

$$
\sum_{\alpha_{1} \in \mathbb{N}} q^{\alpha_{1}}\left(\begin{array}{c}
\alpha_{1} \\
r
\end{array}\right) \sum_{\substack{j=\left|\alpha-\alpha_{1}\right| \\
j \equiv \alpha+\alpha_{1}(\bmod 2)}}^{\alpha+\alpha_{1}} q^{j}=q^{\alpha} T_{q}^{\prime}\left(\left(\begin{array}{c}
\alpha \\
r
\end{array}\right)\right) .
$$

Proof. - Denoting by $\sum$ the left hand side sum of lemma 14, one has

$$
\sum=\frac{q^{\alpha}}{1-q^{2}}\left[\sum_{\alpha_{1} \geqslant \alpha}\left(\begin{array}{c}
\alpha_{1} \\
r
\end{array}\right) q^{2\left(\alpha_{1}-\alpha\right)}+\sum_{\alpha_{1}<\alpha}\left(\begin{array}{c}
\alpha_{1} \\
r
\end{array}\right)-q^{2} \sum_{\alpha_{1}}\left(\begin{array}{c}
\alpha_{1} \\
r
\end{array}\right) q^{2 \alpha_{1}}\right]
$$

The end of the proof is similar to the one of lemma 6 .

We end the section with the following integral expression of $S_{n+2}^{\prime}(0 ; q)$, obtained from proposition 13 and lemma 5 .

LemMA 15. - Let $n$ be a nonnegative integer, then

$$
S_{n+2}^{\prime}(0 ; q)=\frac{\left(1-q^{4}\right)^{n-1}}{\left(1-q^{2}\right)^{3 n}} s_{n-1}\left(\frac{q}{1+q^{2}}\right)
$$

\subsection{Combinatorial expression of the positive moments of $L(f, 1)$.}

In this section, we shall prove the

Proposition 16. - Let $n$ be a nonnegative integer. Then

$$
H_{n+3}=\frac{\zeta(2)^{3 n+3}}{\zeta(4)^{n}} \prod_{p \in \mathcal{P}} s_{n}\left(\frac{p}{p^{2}+1}\right)
$$


By lemma 15 it is equivalent to

Lemma 17. - Let $n$ be a nonnegative integer. Then

$$
H_{n}=\prod_{p \in \mathcal{P}} S_{n}^{\prime}\left(0, \frac{1}{p}\right)
$$

Proof. - By multiplicativity, it suffices to prove

$$
\sum:=\sum_{\nu=0}^{+\infty} \frac{h_{n}\left(p^{\nu}\right)}{p^{\nu}}=S_{n}^{\prime}\left(0, \frac{1}{p}\right)
$$

One has

$$
\sum=\sum_{\substack{\mathbf{b} \in \mathbb{N}^{n} \\ b_{\imath} \mid p \infty(1 \leqslant i \leqslant n)}} \frac{1}{\operatorname{det} \mathbf{b}} \sum_{\substack{\mathbf{d} \in \mathcal{F}^{\prime}(\mathbf{b}) \\ \operatorname{det} \mathbf{d}^{2}=\operatorname{det} \mathbf{b}}} 1 .
$$

Defining $\boldsymbol{\alpha} \in \mathbb{N}^{n}$ by

$$
b_{1}=p^{\alpha_{1}}, \quad \frac{b_{1} \ldots b_{i}}{\left(d_{1} \ldots d_{i-1}\right)^{2}}=p^{\alpha_{\imath}} \quad(2 \leqslant i \leqslant n)
$$

and $\boldsymbol{\beta} \in \mathbb{N}^{n}, \boldsymbol{\delta} \in \mathbb{N}^{n-1}$ by

$$
\begin{aligned}
& b_{i}=p^{\beta_{\imath}}, \quad(1 \leqslant i \leqslant n) \\
& d_{i}=p^{\delta_{\imath}}, \quad(1 \leqslant i \leqslant n-1)
\end{aligned}
$$

one gets

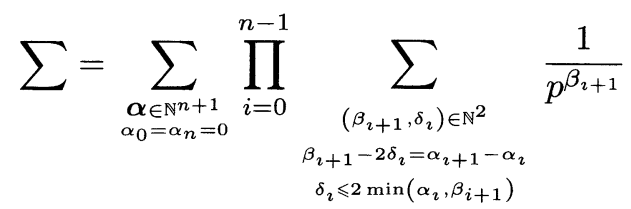

such that it suffices to prove

$$
\sum_{\substack{\left(\beta_{\imath+1}, \delta_{\imath}\right) \in \mathbb{N}^{2} \\ \beta_{\imath+1}-2 \delta_{\imath}=\alpha_{\imath+1}-\alpha_{\imath} \\ \delta_{\imath} \leqslant 2 \min \left(\alpha_{\imath}, \beta_{\imath+1}\right)}} \frac{1}{p^{\beta_{\imath+1}}}=p^{-\left|\alpha_{\imath}-\alpha_{\imath+1}\right|}+p^{-\left|\alpha_{i}-\alpha_{\imath+1}\right|+2}+\ldots+p^{-\left(\alpha_{\imath}+\alpha_{\imath+1}\right)} .
$$

This is done as in the proof of lemma 11. 


\subsection{Asymptotic expansion of the positive moments of $L(f, 1)$.}

In this section, one derives from the combinatorial expression of the positive moments of $L(f, 1)$ the

Proposition 18. - Let $n \geqslant 3$ be an integer, then

$$
\log H_{n}=2 n \log \log n+2 \gamma n+O\left(\frac{n}{\log n}\right)
$$

Proof. - This is a consequence of [Roy03, théorème B and corollaire C] that gives

$n \log \frac{\zeta(2)}{\zeta(4)}+\sum_{p \in \mathcal{P}} \log s_{n}\left(\frac{p}{p^{2}+1}\right)=2 n \log \log n+2 n \log \frac{e^{\gamma}}{\zeta(2)}+O\left(\frac{n}{\log n}\right)$

\section{A unified formula.}

The aim of this section is to give a unified hypergeometrical formula for the moments, positive and negative, of $L(f, 1)$ and $L\left(\operatorname{sym}^{2} f, 1\right)$ proving the proposition $\mathrm{B}$.

Let $a, b$ and $c$ be three complex numbers such that $\mathfrak{R} e c \mathfrak{R} e b>0$, and $z$ a complex number not in the real segment $[1,+\infty[$. One defines

$$
F(a, b, c ; z)=\frac{\Gamma(c)}{\Gamma(b) \Gamma(c-b)} \int_{0}^{1} u^{b-1}(1-u)^{c-b-1}(1-u z)^{-a} \mathrm{~d} u
$$

One has [GR00, 9.131.1]

$$
F(a, b, c ; z)=(1-z)^{c-b-a} F(c-a, c-b, c ; z)
$$


For $\alpha=2$ and $n \leqslant 0$, the result is [Roy03, lemme 23 and théorème B]. For $\alpha=2$ and $n>0$, [Roy03, lemma 23] and proposition 16 gives

$$
\begin{aligned}
& H_{n}= \\
& \prod_{p \in \mathcal{P}}\left(1+\frac{1}{p}\right)^{-2 n-6}\left[1-4 \frac{p}{(p+1)^{2}}\right]^{-n-3 / 2} F\left(-n, \frac{3}{2}, 3 ; 4 \frac{p}{(p+1)^{2}}\right) .
\end{aligned}
$$

The result then follows from (23). For $\alpha=3$ and $n \leqslant 0$, this is [Roy03, théorème $\mathrm{A}$, lemme 16] and (23). For $\alpha=3$ and $n>0$, [Roy03, lemma 16] and proposition 10 give

$$
\begin{aligned}
& M_{n+2}= \\
& \prod_{p \in \mathcal{P}}\left(1-\frac{1}{p}\right)^{-3 n-6}\left[1+4 \frac{p}{(p-1)^{2}}\right]^{-n-3 / 2} F\left(-n, \frac{1}{2}, 2 ;-4 \frac{p}{(p-1)^{2}}\right) .
\end{aligned}
$$

Formula (23) implies the result.

\section{Twisted moments.}

The work done in the preceding sections easily extends to twisted moments, which are defined as follows: for $m \in \mathbb{N}$, put

$$
M_{n}(m):=\lim _{\substack{N \rightarrow+\infty \\ N \in \mathcal{N}_{\text {cri }} \\(N, m)=1}} \sum_{f \in H_{k}^{*}(N)} \omega(f) \lambda_{f}(m) L\left(\operatorname{sym}^{2} f, 1\right)^{n}
$$

where $\lambda_{f}(n)$ is the eigenvalue of the $n$-th Hecke operator. The existence of this limit may be checked as in [Roy01]. As before we find

$$
M_{n}(m)=\zeta(2)^{n} \prod_{p \in \mathcal{P}} S_{n}\left(\frac{v_{p}(m)}{2}, \frac{1}{p}\right)
$$

where $v_{p}(m)$ denotes the $p$-adic valuation of $m$ and

$$
S_{n}(\alpha, q)=0 \quad \text { if } \alpha \notin \mathbb{N} .
$$

One recall the definition

$$
\Sigma_{n}[\alpha](q):=(1-q)^{n}\left(1-q^{2}\right)^{n} S_{n+1}(\alpha ; q)
$$


so that if $\alpha$ is an integer,

$$
\begin{aligned}
\Sigma_{n}[\alpha](q)= & \sum_{\substack{\left(d_{0}, \ldots, d_{n}\right) \in \mathbb{N}^{n+1} \\
d_{0} \leqslant \ldots \leqslant d_{n} \\
d_{i} \leqslant i(0 \leqslant i \leqslant n)}} q^{2 \#\left\{i \in[0, n-1]: d_{i+1}=d_{2}\right\}} \\
& \times(1-q)^{\#\left\{i \in[1, n]: d_{2}=i\right\}}\left(\begin{array}{c}
\alpha \\
n-d_{n}
\end{array}\right) q^{\alpha}\left(q^{-2}-1\right)^{n-d_{n}} .
\end{aligned}
$$

Section 3.1 (and especially equation (13)) then enables us to state the following theorem.

Theorem 19. - Let $n$ be a nonnegative integer. Define

$$
\operatorname{Twist}_{2}(n, m)=\prod_{p \mid m} \frac{\Sigma_{n}\left[v_{p}(m) / 2\right]}{\Sigma_{n}[0]}\left(\frac{1}{p}\right) .
$$

Then

$$
M_{n+1}(m)=\operatorname{Twist}_{2}(n, m) M_{n+1} .
$$

Remark 20. - From

(24)

$$
\begin{aligned}
& \Sigma_{n}[\alpha](q)= \\
& \sum_{D \in \mathcal{D}_{n+1}}(1-q)^{\operatorname{RET}(D)-1} q^{2 \operatorname{DBR}(D)}\left(\frac{1-q^{2}}{q^{2}}\right)^{\operatorname{LD}(D)-1}\left(\begin{array}{c}
\alpha \\
\operatorname{LD}(D)-1
\end{array}\right) q^{\alpha},
\end{aligned}
$$

one deduces the following combinatorial expression

$$
\sum_{\alpha=0}^{+\infty} \Sigma_{n}[\alpha](q) t^{\alpha}=\frac{q}{(1-q)\left(1-q^{2}\right) t} K_{n+1}\left(1-q, q^{2}, \frac{\left(1-q^{2}\right) t}{q(1-q t)}\right)
$$

where $K_{n}$ has been defined in section 2 . Note that the value at $t=0$ of the left hand side of $(25)$ is $\Sigma_{n}[0](q)$, which by definition is given by

$$
\begin{aligned}
\Sigma_{n}[0](q) & =\sum_{\substack{D \in \mathcal{D}_{n}+1 \\
\mathrm{LD}(D)=1}}(1-q)^{\operatorname{RET}(D)-1} q^{2 \operatorname{DBR}(D)} \\
& =\left.\frac{1}{1-q} \frac{\partial}{\partial z} K_{n+1}\left(1-q, q^{2}, z\right)\right|_{z=0} .
\end{aligned}
$$


This is also the right hand side of $(25)$ since $K_{n+1}\left(1-q, q^{2}, 0\right)=0$ (the only path with LD $=0$ being the empty one).

Remark 21. - Since there is a bijection

$$
\varphi: \mathcal{D}_{n}^{*}:=\left\{D \in \mathcal{D}_{n+1}: \operatorname{LD}(D)=1\right\} \stackrel{\sim}{\longrightarrow} \mathcal{D}_{n}
$$

with $\operatorname{RET}(\varphi(D))=\operatorname{RET}(D)-1$ and $\operatorname{DBR}(\varphi(D))=\operatorname{DBR}(D)$ (remove the last two steps of paths of $\mathcal{D}_{n}^{*}$ ), one recovers by combinatoric means the value of $M_{n+1}$ :

$$
M_{n+1}=\zeta(2)^{n+1} \prod_{p \in \mathcal{P}}\left(1-\frac{1}{p}\right)^{-n}\left(1-\frac{1}{p^{2}}\right)^{-n} \Sigma_{n}[0]\left(\frac{1}{p}\right)
$$

where

$$
\begin{aligned}
\Sigma_{n}[0](q) & =\sum_{\substack{D \in \mathcal{D}_{n}+1 \\
\mathrm{LD}(D)=1}}(1-q)^{\mathrm{RET}(D)-1} q^{2 \mathrm{DBR}(D)} \\
& =\sum_{D \in \mathcal{D}_{n}}(1-q)^{\operatorname{RET}(D)} q^{2 \mathrm{DBR}(D)}=A_{n}\left(1-q, q^{2}\right) .
\end{aligned}
$$

It has to be compared with lemma 11 and corollary 8.

Remark 22. - One interprets theorem 19 in terms of independance of random variables. From

$$
\omega(f)=\frac{1}{\# H_{k}^{*}(N)} \frac{\zeta(2)}{L\left(\operatorname{sym}^{2} f, 1\right)}\left[1+O_{k}\left(\frac{1}{\log \log (3 N)}\right)\right]
$$

for $N \in \mathcal{N}_{\text {cri }}$ (see $[$ RW04, (16) and (30)]) one deduces

$$
\lim _{\substack{N \rightarrow+\infty \\ N \in \mathcal{N}_{\text {cri }} \\(N, m)=1}} \frac{1}{\# H_{k}^{*}(N)} \sum_{f \in H_{k}^{*}(N)} \lambda_{f}(m) L\left(\operatorname{sym}^{2} f, 1\right)^{n}=\zeta(2) M_{n+1}(m) .
$$

In particular (take $m=1) \zeta(2) M_{n+1}$ is the moment of order $n$ of the limit of the random variable $f \mapsto L\left(\operatorname{sym}^{2} f, 1\right)$. One then describes the moments of the limit of the random variable $f \mapsto \lambda_{f}(m)$. Denote by $X_{r}$ is the $r$-th Chebyshev polynomial of second kind, defined by

$$
X_{r}(2 \cos \varphi)=\frac{\sin [(r+1) \varphi]}{\sin (\varphi)} \quad(\varphi \in[0, \pi]) .
$$


The basis $\left\{X_{r}\right\}_{r \in \mathbb{N}}$ is orthonormal for the scalar product on $\mathbb{R}[X]$ defined by the Sato-Tate measure

$$
\langle P, Q\rangle:=\int_{-2}^{2} P(x) Q(x) \mu_{\infty}(x), \quad \mu_{\infty}(x)=\frac{1}{\pi} \sqrt{1-\frac{x^{2}}{4}} \mathrm{~d} x .
$$

A direct computation then leads to the decomposition

$$
X^{r}=\sum_{j=0}^{r} x(r, j) X_{j}
$$

with

$$
x(r, j)=\delta(2 \mid j+r) \frac{2^{r+2}}{\pi} \int_{0}^{\pi / 2} \cos (\varphi)^{r} \sin [(j+1) \varphi] \sin (\varphi) \mathrm{d} \varphi .
$$

The multiplicativity of Hecke operators gives

$$
\begin{aligned}
& \lim _{\substack{N \rightarrow+\infty \\
N \in \mathcal{N}_{\text {cri }} \\
(N, m)=1}} \frac{1}{\# H_{k}^{*}(N)} \sum_{f \in H_{k}^{*}(N)} \lambda_{f}(m)^{r} L\left(\operatorname{sym}^{2} f, 1\right)^{n} \\
& =\zeta(2) \sum_{j=0}^{r} x(r, j) \operatorname{Twist}_{2}\left(n, m^{j}\right) M_{n+1} .
\end{aligned}
$$

Denote by $\mathbf{1}^{\square}$ the characteristic function of squares and by $\mu_{m}$ the measure on $[-2,2]$ given by

$$
\mu_{m}(x)=\frac{m+1}{\left(m^{1 / 2}+m^{-1 / 2}\right)^{2}-x^{2}} \mu_{\infty}(x) .
$$

Then Selberg trace formula (see [ILS00, propositions 2.11 and 2.13]) leads to

$$
\begin{aligned}
\lim _{\substack{N \rightarrow+\infty \\
N \in \mathcal{N}_{\text {cri }} \\
(N, m)=1}} \frac{1}{\# H_{k}^{*}(N)} \sum_{f \in H_{k}^{*}(N)} \lambda_{f}(m)^{r} & =\sum_{j=0}^{r} x(r, j) \frac{\mathbf{1}^{\square}\left(m^{j}\right)}{m^{j / 2}} \\
& =\sum_{j=0}^{r} x(r, j) \int_{-2}^{2} X_{j}(x) \mu_{m}(x) \\
& =\int_{-2}^{2} x^{r} \mu_{m}(x)
\end{aligned}
$$


for all $r \geqslant 1$, so that $\mu_{m}$ is the equirepartition measure of the family

$$
\bigcup_{\substack{N \in \mathcal{N}_{\text {cri }} \\(N, m)=1}}\left\{\lambda_{f}(m): f \in H_{k}^{*}(N)\right\}
$$

(see also [Ser97]). By (27) and (28), the system of equalities

$$
\sum_{j=0}^{r} x(r, j) \operatorname{Twist}_{2}\left(n, m^{j}\right)=\sum_{j=0}^{r} x(r, j) \frac{\mathbf{1}^{\square}\left(m^{j}\right)}{m^{j / 2}}, \quad(r, n) \in \mathbb{N}^{2}
$$

is equivalent to the independance of the limits of the random variables

$$
f \mapsto \lambda_{f}(m) \quad \text { and } \quad f \mapsto L\left(\operatorname{sym}^{2} f, 1\right) .
$$

Remark 23. - The coefficient Twist $2(n, m)$ is zero if and only if $m$ is not a squarefull integer (see (24)). Assume $m$ is a squarefull integer. One proves that $\lambda_{f}(m)$ does not affect the asymptotic behavior of the moments of $L\left(\operatorname{sym}^{2} f, 1\right)$. Let $\alpha \in \mathbb{N}$ and $\left.q \in\right] 0,1[$. Equation (24) implies

$$
\Sigma_{n}[\alpha](q) \geqslant \sum_{\substack{D \in \mathcal{D}_{n+1} \\ \text { LD }(D)=1}}(1-q)^{\operatorname{RET}(D)-1} q^{2 \operatorname{DBR}(D)} q^{\alpha}
$$

so that (26) gives

$$
\Sigma_{n}[\alpha](q) \geqslant q^{\alpha} \Sigma_{n}[0](q)
$$

In the proof of lemma 9 , one introduced the function

$$
F_{n}(q)=\frac{1}{1-q} \Sigma_{n}[0](q)
$$

and proved the integral representation

$$
F_{n}(q)=\frac{4}{\pi} \int_{0}^{\pi / 2}\left[(1-q)^{2}+4 q \sin ^{2} \theta\right]^{n} \cos ^{2} \theta \mathrm{d} \theta .
$$

By (24), one has the majoration

$$
\begin{aligned}
\Sigma_{n}[\alpha](q) & \leqslant \sum_{D \in \mathcal{D}_{n+1}}(1-q)^{\operatorname{RET}(D)-1} q^{2 \operatorname{DBR}(D)}\left[\sum_{\ell \in \mathbb{Z}}\left(\begin{array}{l}
\alpha \\
\ell
\end{array}\right)\left(\frac{1-q^{2}}{q^{2}}\right)^{\ell} q^{\alpha}\right] \\
& \leqslant \frac{1}{q^{\alpha}} \sum_{D \in \mathcal{D}_{n+1}}(1-q)^{\operatorname{RET}(D)-1} q^{\operatorname{DBR}(D)} .
\end{aligned}
$$


Bijection $\varphi$ - see remark 21 - enables to write

$$
\sum_{D \in \mathcal{D}_{n+1}}(1-q)^{\operatorname{RET}(D)-1} q^{2 \operatorname{DBR}(D)}=(1-q) \sum_{\substack{D \in \mathcal{D}_{n+2} \\ \text { LD }(D)=1}}(1-q)^{\operatorname{RET}(D)-1} q^{2 \mathrm{DBR}(D)}
$$

so that (26) gives

$$
\Sigma_{n}[\alpha](q) \leqslant \frac{1-q}{q^{\alpha}} \Sigma_{n+1}[0](q) .
$$

Using (30) and (31) one shows

$$
\Sigma_{n+1}[0](q) \leqslant(1+q)^{2} \Sigma_{n}[0](q)
$$

thus

$$
\Sigma_{n}[\alpha](q) \leqslant \frac{(1-q)(1+q)^{2}}{q^{\alpha}} \Sigma_{n}[0](q) \leqslant \frac{1}{q^{\alpha+1}} \Sigma_{n}[0](q)
$$

Equations (29) and (32) then imply

$$
\frac{1}{\sqrt{m}} \leqslant \operatorname{Twist}_{2}(n, m) \leqslant m \quad(m \text { squarefull }) .
$$

Finally

$$
\log M_{n}(m)=3 n \log \log n+3 \gamma n+O\left(\frac{n}{\log n}+\log m\right) \quad(m \text { squarefull }) .
$$

Similarly we also define

$$
H_{n}(m):=\lim _{\substack{N \rightarrow+\infty \\ N \in \mathcal{N}_{\text {cri }} \\(m, N)=1}} \sum_{f \in H_{k}^{*}(N)} \omega(f) \lambda_{f}(m) L(f, 1)^{n} .
$$

We have the counterpart of theorem 19. Recall the definition

$$
\begin{aligned}
\sigma_{n}[\alpha](q):= & \sum_{\substack{\left(d_{0}, \ldots, d_{n}\right) \in \mathbb{N}^{n+1} \\
d_{0} \leqslant \ldots \leqslant d_{n} \\
d_{i} \leqslant i(0 \leqslant i \leqslant n)}} q^{2\left(\#\left\{i \in[0, n-1]: d_{\imath+1}=d_{i}\right\}-n+d_{n}\right)} \\
& \times\left(1-q^{2}\right)^{\#\left\{i \in[1, n]: d_{r}=i\right\}+n-d_{n}}\left(\begin{array}{c}
\alpha \\
n-d_{n}
\end{array}\right) q^{\alpha}
\end{aligned}
$$


Theorem 24. - Let $n$ be a nonnegative integer. Define

$$
\operatorname{Twist}_{1}(n, m)=\prod_{p \mid m} \frac{\sigma_{n}\left[v_{p}(m)\right]}{\sigma_{n}[0]}\left(\frac{1}{p}\right)
$$

Then

$$
H_{n+1}(m)=\operatorname{Twist}_{1}(n, m) H_{n+1} .
$$

Remark 25. - From

$$
\begin{aligned}
& \sigma_{n}[\alpha](q) \\
& =\sum_{D \in \mathcal{D}_{n+1}}\left(1-q^{2}\right)^{\operatorname{RET}(D)-1} q^{2 \operatorname{DBR}(D)}\left(\frac{1-q^{2}}{q^{2}}\right)^{\operatorname{LD}(D)-1}\left(\begin{array}{c}
\alpha \\
\operatorname{LD}(D)-1
\end{array}\right) q^{\alpha},
\end{aligned}
$$

one deduces the following combinatorial expression

$$
\sum_{\alpha=0}^{+\infty} \sigma_{n}[\alpha](q) t^{\alpha}=\frac{q}{\left(1-q^{2}\right)^{2} t} K_{n+1}\left(1-q^{2}, q^{2}, \frac{\left(1-q^{2}\right) t}{q(1-q t)}\right)
$$

where $K_{n}$ has been defined in section 2 .

Remark 26. - Similary to (27) one has

$$
\lim _{\substack{N \rightarrow+\infty \\ N \in \mathcal{N}_{\text {cri }} \\(N, m)=1}} \sum_{f \in H_{k}^{*}(N)} \omega(f) \lambda_{f}(m)^{r} L(f, 1)^{n+1}=\sum_{j=0}^{r} x(r, j) \operatorname{Twist}_{1}\left(n, m^{j}\right) H_{n+1} .
$$

Then, for $m>1$, Petersson trace formula leads to

$$
\lim _{\substack{N \rightarrow+\infty \\ N \in \mathcal{N}_{\text {cri }} \\(N, m)=1}} \sum_{f \in H_{k}^{*}(N)} \omega(f) \lambda_{f}(m)^{r}=x(r, 0) .
$$

In the probability space where a form $f$ has weight $\omega(f)$, the independance of the limit random variables

$$
f \mapsto \lambda_{f}(m) \quad \text { and } \quad f \mapsto L(f, 1)
$$


is equivalent to the system of equalities

$$
\sum_{j=0}^{r} x(r, j) \operatorname{Twist}_{1}\left(n, m^{j}\right)=x(r, 0), \quad(r, n) \in \mathbb{N}^{2} .
$$

Similary to (29) and (32) and using

$$
\sigma_{n}[0](q)=\frac{1}{1-q^{2}} N_{n-1}(q)
$$

one has

$$
q^{\alpha} \sigma_{n}[0](q) \leqslant \sigma_{n}[\alpha](q) \leqslant \frac{\left(1-q^{2}\right)(1+q)^{2}}{q^{\alpha}} \sigma_{n}[0](q) \leqslant \frac{\sigma_{n}[0](q)}{q^{\alpha+1}}
$$

so that

$$
\frac{1}{\sqrt{m}} \leqslant \operatorname{Twist}_{1}(n, m) \leqslant m
$$

and

$$
\log H_{n}(m)=2 n \log \log n+2 \gamma n+O\left(\frac{n}{\log n}+\log m\right) .
$$

\section{BIBLIOGRAPHY}

[Cho34] S. CHowla, On the k-analogue of a result in the theory of the Riemann zeta function, Math. Z. 38, 483-487 (1934).

[CM04] J. Cogdell, P. Michel, On the Complex Moments of Symmetric power $L$ functions at $s=1$, Int. Math. Res. Not., $\mathrm{n}^{\circ}$. 31, 1561-1617 (2004). MR 2 035301

[Del03] C. Delaunay, Computing the Modular Degree of an Elliptic Curve using L-functions, J. Théor. Nombres Bordeaux 15, $\mathrm{n}^{\circ} .3$ (2003).

[GR00] I.S. GRADShTEYN, I.M. RYZHIK, Table of integrals, series, and products, sixth ed., Academic Press Inc., San Diego, CA, 2000, Translated from the Russian, Translation edited and with a preface by Alan Jeffrey and Daniel Zwillinger. MR 2001c:00002

[GS02] A. Granville, K. Soundararajan, Upper bounds for $|L(1, \chi)|$, Q. J. Math. 53, $\mathrm{n}^{\circ} .3,265-284$ (2002). MR 2003h:11104

[ILS00] H. IWANIEC, W. LuO, P. SARNAK, Low lying zeros of families of $L$-functions, Inst. Hautes Études Sci. Publ. Math., n. 91, 55-131 (2001). MR 2002h:11081

[Luo99] W. Luo, Values of symmetric square $L$-functions at 1, J. Reine Angew. Math. 506, 215-235 (1999). MR 2001d:11055 
[MV99] H.L. MONTGOMERY, R.C. VAUGhan, Extreme values of Dirichlet $L$-functions at 1 , Number theory in progress, Vol. 2 (Zakopane-Kościelisko, 1997), de Gruyter, Berlin, 1039-1052 (1999). MR 2000m:11075

[Roy01] E. RoYER, Statistique de la variable aléatoire $L\left(\operatorname{sym}^{2} f, 1\right)$, Math. Ann. 321, $\mathrm{n}^{\circ}$. 3, 667-687 (2001). MR 1871974

[Roy03] E. ROYER, Interprétation combinatoire des moments négatifs des valeurs de fonctions $L$ au bord de la bande critique, Annales scientifiques de l'École Normale Supérieure 36 (2003), $\mathrm{n}^{\circ}$. 4, 601-620. MR 2013928

[RW04] E. ROYER, J. WU, Taille des valeurs de fonctions $L$ de carrés symétriques au bord de la bande critique, Rev. Mat. Iberoamericana $(\geqslant 2004)$, To appear. Available at http://www.carva.org/emmanuel.royer.

[Ser97] J.-P. SERRE, Répartition asymptotique des valeurs propres de l'opérateur de Hecke $T_{p}$, J. Amer. Math. Soc. 10, n . 1, 75-102 (1997). MR 97h:11048

[Sul98] R.A. SulANKE, Catalan path statistics having the Narayana distribution, Proceedings of the 7th Conference on Formal Power Series and Algebraic Combinatorics (Noisy-le-Grand, 1995), vol. 180, 369-389 (1998). MR 99b:05004

[Ten95] G. Tenenbaum, Introduction à la théorie analytique et probabiliste des nombres, second ed., Cours Spécialisés [Specialized Courses], vol. 1, Société Mathématique de France, Paris (1995). MR 97e:11005a

[Wat02] M. Watkins, Computing the modular degree of an elliptic curve, Experiment. Math. 11 (2002), nº.4, 487-502 (2003). MR 1969641

Manuscrit reçu le 2 mars 2004, accepté le 8 juin 2004 .

Laurent HABSIEGER, Université Claude Bernard Lyon I Institut Girard Desargues CNRS UMR 5028

43, boulevard du 11 novembre 1918 69622 Villeurbanne Cedex (France)

habsiege@euler.univ-lyon1.fr

Emmanuel ROYER, Université Paul Valéry Montpellier III MIAp 34199 Montpellier cedex 5 (France)

Université Montpellier II I3M - UMR CNRS 5149 CC 051 34095 Montpellier cedex 5 (France) emmanuel.royer@polytechnique.org royer@darboux.math.univ-montp2.fr 